\title{
Conservation and divergence of ADAM family proteins in the Xenopus genome
}

\author{
Shuo Wei*1, Charles A Whittaker2, Guofeng X ${ }^{1}$, Lance C Bridges ${ }^{1,3}$, Anoop Shah'1, Judith M White ${ }^{1}$ and \\ Douglas W DeSimone ${ }^{1}$
}

\begin{abstract}
Background: Members of the disintegrin metalloproteinase (ADAM) family play important roles in cellular and developmental processes through their functions as proteases and/or binding partners for other proteins. The amphibian Xenopus has long been used as a model for early vertebrate development, but genome-wide analyses for large gene families were not possible until the recent completion of the $X$. tropicalis genome sequence and the availability of large scale expression sequence tag (EST) databases. In this study we carried out a systematic analysis of the $X$. tropicalis genome and uncovered several interesting features of ADAM genes in this species.

Results: Based on the $X$. tropicalis genome sequence and EST databases, we identified Xenopus orthologues of mammalian ADAMs and obtained full-length cDNA clones for these genes. The deduced protein sequences, synteny and exon-intron boundaries are conserved between most human and $X$. tropicalis orthologues. The alternative splicing patterns of certain Xenopus ADAM genes, such as adams 22 and 28, are similar to those of their mammalian orthologues. However, we were unable to identify an orthologue for ADAM7 or 8. The Xenopus orthologue of ADAM15, an active metalloproteinase in mammals, does not contain the conserved zinc-binding motif and is hence considered proteolytically inactive. We also found evidence for gain of ADAM genes in Xenopus as compared to other species. There is a homologue of ADAM10 in Xenopus that is missing in most mammals. Furthermore, a single scaffold of $X$. tropicalis genome contains four genes encoding ADAM28 homologues, suggesting genome duplication in this region.

Conclusions: Our genome-wide analysis of ADAM genes in X. tropicalis revealed both conservation and evolutionary divergence of these genes in this amphibian species. On the one hand, all ADAMs implicated in normal development and health in other species are conserved in $X$. tropicalis. On the other hand, some ADAM genes and ADAM protease activities are absent, while other novel ADAM proteins in this species are predicted by this study. The conservation and unique divergence of ADAM genes in Xenopus probably reflect the particular selective pressures these amphibian species faced during evolution.
\end{abstract}

\section{Background}

ADAMs belong to the M12B subfamily of metalloproteinases and metalloproteinase-like proteins [1]. A prototype ADAM is a type I transmembrane protein, but some ADAMs are also present as soluble forms, either due to alternative splicing or protease-mediated cleavage ("shedding") from the cell surface [2,3]. ADAMs are multidomain proteins with an extracellular metalloproteinase domain, a disintegrin domain and a cysteine-rich domain; therefore they are also called MDC (metalloproteinase/

*Correspondence: sw9hy@virginia.edu

1 Department of Cell Biology and the Morphogenesis and Regenerative Medicine Institute, University of Virginia, Charlottesville, VA 22908, USA Full list of author information is available at the end of the article disintegrin/cysteine-rich) proteins. Some but not all ADAMs contain a canonical zinc-binding motif within the metalloproteinase domain, which is required for protease activity $[2,3]$. The disintegrin domain can selectively interact with different integrins [4]; together with the cysteine-rich domain, it may modulate cell-cell and cellmatrix adhesion [4-6], as well as substrate recognition by the metalloproteinase domain $[7,8]$. The cytoplasmic tail contains binding sites for a variety of cellular proteins, and may be involved in inside-out signaling that regulates the activity of the ectodomain [9-11].

A phylogenetic tree of ADAMs identified in different species can be found in the tree families database TreeFam [12]. About half of the ADAMs are solely or predom- 
inantly expressed in the testis of mammals (i.e. testisspecific), with no orthologue found in nonmammalian species $[12,13]$. Functions of these ADAMs are thus mainly related to mammalian reproduction. The other ADAMs are expressed widely in tissues and organs other than the testis. Many of these ADAMs are implicated in embryonic development. Mice lacking or carrying defective ADAM genes often display developmental abnormalities that vary from defects in adipogenesis and myogenesis [14] and mild dysfuctions in the central nervous system $(\mathrm{CNS})[15,16]$, to more severe defects such as early embryonic and perinatal lethality [17-19]. The activities of ADAMs are also linked to a variety of human diseases, such as cancer [20] and cardiovascular diseases [21], as well as rheumatoid arthritis and other inflammatory diseases [22]. However, the molecular bases for the functions of ADAMs are largely unappreciated, and identifying the in vivo substrates and binding partners for ADAMs remains a major challenge $[2,23]$. Furthermore, functional redundancy between two or more ADAMs seems to be a common phenomenon [24,25], posing an additional difficulty for understanding the roles of ADAMs in development and diseases.

One area that has drawn increasing interest concerns the roles of ADAMs in cell signaling, mainly via shedding of cell surface proteins by ADAM metalloproteinase activities. Through this process, ADAMs release key extracellular signaling proteins (e.g. growth factors such as EGF and cytokines such as TNF- $\alpha$ ) from the cell surface $[3,22]$. ADAMs also participate in the generation of key intracellular signals (e.g. Notch and ErbB4 intracellular domain) by performing an obligate ectodomain cleavage event prior to a regulated intramembraneous proteolytic cleavage [26,27]. Recently, a subgroup of nonproteolytic ADAMs, ADAMs 11, 22 and 23, were shown to function as receptors for the secreted Leucine-rich glioma inactivated (LGI) proteins, which are required for proper synaptic transmission in the brain $[28,29]$. Regulation of cell adhesion and migration is another major function of ADAMs. A large body of evidence suggests that ADAM disintegrin domains can interact with integrins, although most ADAMs do not contain the conserved RGD integrin binding sequence [30,31]. The cysteinerich domain of ADAM12 can also mediate cell adhesion by binding cell surface syndecans [6,32]. Furthermore, ADAM metalloproteinase activities are also involved in remodeling extracellular matrix and regulating cell-cell adhesion through cleavage of extracellular matrix components (e.g. type IV collagen and fibronectin) [33-35] and adhesion molecules (e.g. cadherins) [36-40].

The amphibian Xenopus laevis has been used for decades as a model system for studying early vertebrate development. The easy availability of large numbers of fertilized eggs, the ability of the embryos to develop and be manipulated in vitro, and the transparent epidermis of the tadpoles provide some practical advantages over mammalian and other models. However, the pseudo-tetraploid genome and long generation time of this species prevented it from being widely used for genetic and genomic research. These difficulties were overcome in large part by the adoption of Xenopus tropicalis, a closely related species with a true diploid genome and a relatively short generation time. The genome sequence of $X$. tropicalis is now complete, and large collections of EST clones from both X. tropicalis and X. laevis are available, providing useful tools for systematic prediction and characterization of large families of genes. A recently published genome-wide analysis of the matrix metalloproteinases (MMPs), another family of extracellular metalloproteinases that is related to the ADAMs, revealed evolutionary conservation and distinct duplication patterns of MMPs in X. tropicalis as compared to humans and other mammals [41]. Several ADAM genes have been cloned previously from X. laevis and characterized for their functions in fertilization and development [34,42-45]. Given the key roles of these ADAMs in vivo, it is important to establish their orthologues in mammals and to identify their paralogues in the Xenopus genome.

As an initial step for systematic studies of all ADAM family proteins in the frog species, we started out to identify the homologues of known vertebrate ADAMs that are expressed in $X$. tropicalis. Our study shows that most non-testis specific ADAMs are conserved between frogs and mammals. However, some potential loss or gain of ADAM genes (and possibly functions) in X. tropicalis as compared to mammals suggests evolutionary divergence of ADAMs in this amphibian species.

\section{Results and discussion ADAM genes in the $X$. tropicalis genome}

Searches of the current version (v4.1) of the $X$. tropicalis genome assembly yielded homologues for most known vertebrate non-testis specific ADAMs. Sequences of these predicted ADAM genes were then used to search against the EST databases (or to design primers for experimental cloning) to obtain full-length cDNA clones from $X$. tropicalis and, where possible, $X$. laevis. No orthologues of the mammalian testis-specific ADAMs have been found in any nonmammalian species [12,13]; similarly, we were unable to identify a Xenopus orthologue for any of these ADAMs. A predicted gene encoding the orthologue of $X$. laevis ADAM16 (previously known as $x M D C 16)$, an ADAM expressed only in testis with a potential role in fertilization [42], was localized to Scaffold_40 of $X$. tropicalis genome. No orthologue of ADAM16 was found in any other species examined, 
including mammals, fish and chicken. Therefore it is likely that ADAM16 function is limited to reproduction of the frogs and related species.

A phylogenetic tree of $X$. tropicalis and human non-testis specific ADAMs is shown in Figure 1A, in which these ADAMs are divided into several subfamilies ("clades"). Results of this analysis are consistent with an expanded tree including other representative vertebrate species (Additional File 1), and with previously published phylogenetic studies of ADAMs from various species $[12,30,46]$. Comparison of the exon-intron structures of $X$. tropicalis ADAM genes with those of human ADAM genes [46] reveals conservation of most of the splice sites between orthologues (Figure 1B). ADAMs can also be divided into clades based on their splicing patterns, as suggested by Kleino et al. ([46] and Figure 1B). While this subgrouping method places emphasis on conservation and divergence in gene structure, the method used in Figure $1 \mathrm{~A}$ is based on sequence similarity and probably better reflects the conservation and evolution in gene function. In general the phylogeny-based subgrouping (Figure 1A) agrees well with the splicing-based method ([46] and Figure 1B), but it also differs from the latter in two aspects: the exclusion of ADAM9 from the meltrin clade (ADAMs 12, 13/33 and 19) and the inclusion of ADAMs 10 and 17 into the same clade. We believe that the former (Figure 1A) better indicates the functional relationship among ADAM paralogues (as discussed in the following sections). The phylogeny-based subgrouping is the method of choice here because sequence homology and gene functions are the primary focus of this study.

Sequence information and expression patterns of identified Xenopus ADAM genes are summarized in Table 1. Several ADAMs, including ADAMs 9, 10, 11, 12, 17, 19, 22 and 23, are known to be required for normal development and health in mice [14-19,47-50]. As expected, all of these essential ADAMs are conserved in frogs, with $>50 \%$ identities in protein sequence between $X$. tropicalis and human orthologues; the other ADAMs are more divergent (Table 1). In cases where full-length sequence information of $X$. laevis cDNA is also available, the similarities between $X$. tropicalis and $X$. laevis orthologues are high ( $>80 \%$ identities in protein sequence), consistent with the close relationship between these two species (Table 1).

The orthologous relationship between human and $X$. tropicalis ADAMs is further supported by synteny analyses, which show the conservation of genetic linkages between most adams and nearby genes in these two species (Figure 2). However, we were unable to identify any likely orthologue for ADAM7 or 8 in the $X$. tropicalis genome or in $X$. tropicalis/X. laevis EST databases. ADAM7 is mainly expressed in the testis [51], but limited expression is also detected in muscles and kidney [52].
A

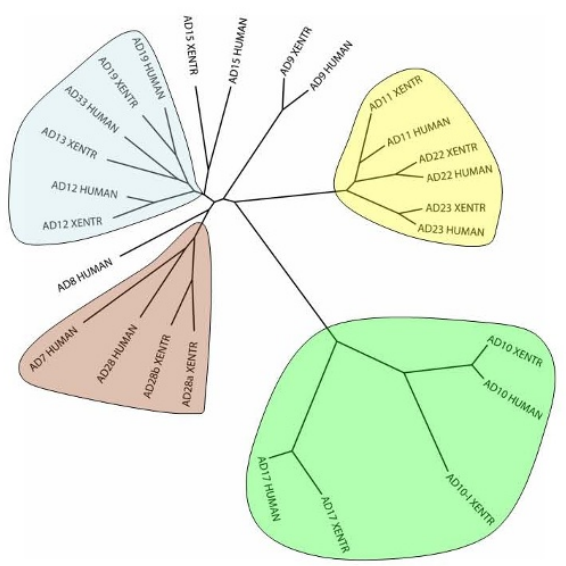

B

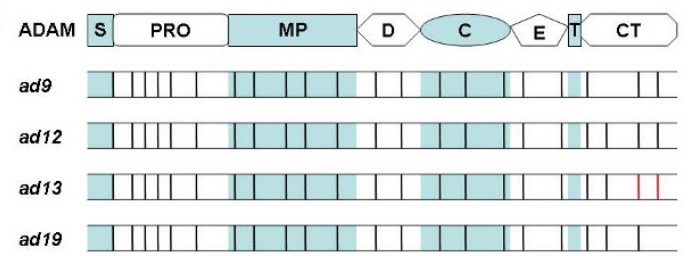
ad15 प||l||||||||||||||

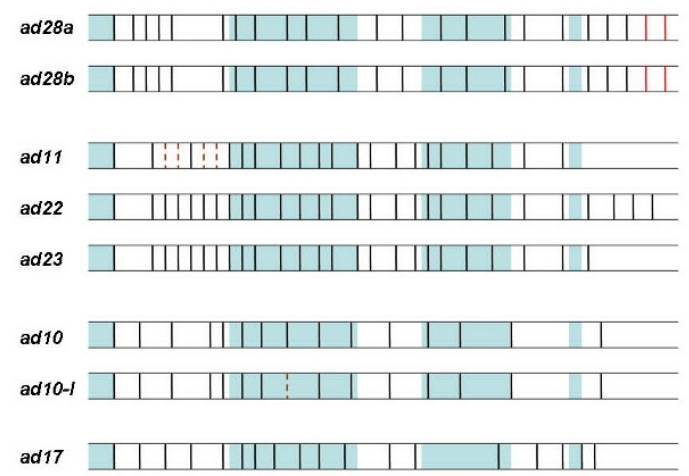

Figure 1 Phylogenetic and splicing analyses of human and $X$. tropicalis ADAMs. A) Phylogenetic tree of human and $X$. tropicalis ADAMs. Protein sequences of human and $X$. tropicalis (XENTR) ADAMs were aligned, and a neighbor-joining tree was drawn using ClustalX. Clades are highlighted by different colors. See Additional File 1 for an expanded phylogenetic tree including other representative vertebrate species, and Additional File 6, A-C for trees generated using alternative models. B) Comparison of splicing patterns of human and $X$. tropicalis adam transcripts. Splice sites within different domains (as indicated at top) of $X$. tropicalis adam genes, as compared with their human orthologues, are shown. Human adams 33 and 10 were used in the comparison with X. tropicalis adams 13 and 10-like (ad10-l), respectively. Black vertical lines represent splice sites that are conserved between these two species, and solid and dotted red vertical lines represent splice sites that are used by $X$. tropicalis adams or their human orthologues, respectively, but not both. Adam genes are divided into different subgroups (adams 9/12/13/19, 15,28a/b, 11/22/23, 10/10-/and 17) based on their splicing patterns, as in Ref. 46. S, signal peptide; $P R O$, propeptide; MP, metalloproteinase domain; D, disintegrin domain; C, cysteine-rich domain; E, EGF-like domain; $T$, transmembrane region; $C T$, cytoplasmic tail. 
Table 1: X. tropicalis ADAM genes identified in this study

\begin{tabular}{|c|c|c|c|c|}
\hline Encoded ADAM & GenBank accession & $\begin{array}{l}\text { \% identity with closest } \\
\text { human homologue }\end{array}$ & $\begin{array}{c}\text { \% identity with } \\
x . \text { laevis orthologue }\end{array}$ & Expression pattern* \\
\hline ADAM9 & $\underline{\mathrm{HM} 467820}$ & 69 & 91 & Widespread \\
\hline ADAM10 & $\underline{\mathrm{DO} 287908}$ & 82 & 93 & Widespread \\
\hline ADAM10-like & $\underline{\mathrm{HM} 483366}$ & 43 (with ADAM10) & ND & Widespread \\
\hline ADAM $11^{\dagger}$ & $\underline{\mathrm{HM} 467821}$ & 76 & 95 & $\begin{array}{l}\text { Tailbud stage head; tadpole; metamorphic tail; } \\
\text { adult brain (high level) }\end{array}$ \\
\hline ADAM12 & $\underline{\text { DQ393787 }}$ & 66 & ND & Gastrula; neurula; tadpole \\
\hline ADAM13/33 & $\underline{\mathrm{DQ} 393788}$ & 53 (with ADAM33) & 91 & $\begin{array}{l}\text { Gastrula; neurula; tadpole; metamorphic tail; adult } \\
\text { spleen }\end{array}$ \\
\hline ADAM15 ${ }^{+}$ & $\underline{\mathrm{HM} 467822}$ & 44 & 81 & $\begin{array}{l}\text { Gastrula; metamorphic limb; adult brain, lung, } \\
\text { testis }\end{array}$ \\
\hline ADAM17 & $\underline{\mathrm{HM} 483367}$ & 68 & 91 & Neurula; adult brain, testis, oviduct \\
\hline ADAM19 & DQ393789 & 59 & 85 & Gastrula; neurula; tadpole \\
\hline ADAM $22^{\dagger \Delta}$ & $\begin{array}{l}\frac{\mathrm{HM} 467823}{\mathrm{HM} 467824} \\
\underline{\mathrm{HM} 467825} \\
\end{array}$ & 71 & 83 & $\begin{array}{l}\text { Tadpole; metamorphic brain/spinal cord; adult } \\
\text { brain (high level), testis }\end{array}$ \\
\hline${\text { ADAM } 23^{+}}^{+}$ & $\underline{\mathrm{HM} 467826}$ & 72 & ND & $\begin{array}{l}\text { Tadpole; adult brain (high level), spleen, kidney, } \\
\text { thymus }\end{array}$ \\
\hline ADAM28a $a^{\ddagger}$ & $\underline{\text { HM} 467827}$ & 47 & 82 & Widespread \\
\hline ADAM28b $\mathrm{b}^{\ddagger}$ & $\underline{\mathrm{HM} 467828}$ & 44 & ND & Neurula; tadpole; adult spleen, bone, kidney \\
\hline
\end{tabular}

* Expression patterns were estimated by the presence of ESTs (full or partial) in different organs and/or developmental stages, as deposited in GenBank. "Widespread" was assigned when ESTs are present in multiple (>20) organs, and "high level" was assigned when multiple (>20) EST entries are present in a single organ at a single developmental stage and account for $>50 \%$ of all ESTs found in GenBank.

${ }^{\dagger}$ ADAMs predicted to be proteolytically inactive.

₹ Two additional adam28 homologues ( $a d a m 28 \mathrm{c}$ and $d$ ) were identified on Scaffold 30, but full-length cDNA clones have not been obtained. $\triangle$ Three alternatively spliced isoforms of $X$. tropicalis ADAM22 were identified in this study. ISO1 ( HM467823 was used to calculate sequence identities here.

ND: not determined.

This ADAM seems to be mammal-specific and no orthologue has been identified to date in other animals [12]. Therefore it is perhaps not surprising that it is also absent in frogs. In the human genome adam7 lies next to adam 28 and adamdec1, whereas in X. tropicalis this metalloproteinase gene "cluster" is replaced by four homologues of adam 28 in tandem (Figure 2K; see further discussion below). ADAM8 is one of the most widely studied ADAMs, with potential roles in cancer and inflammatory diseases [53]. However, mice with targeted deletion of the ADAM8 gene do not display any apparent developmental or pathological phenotypes [54], indicating that this gene is dispensable for normal development and health. The human adam 8 gene was mapped to chromosome 10, with tubgcp 2 and znf511 on one side and $k n d c 1$ and gpr123 on the other side (Figure 2L). Although 


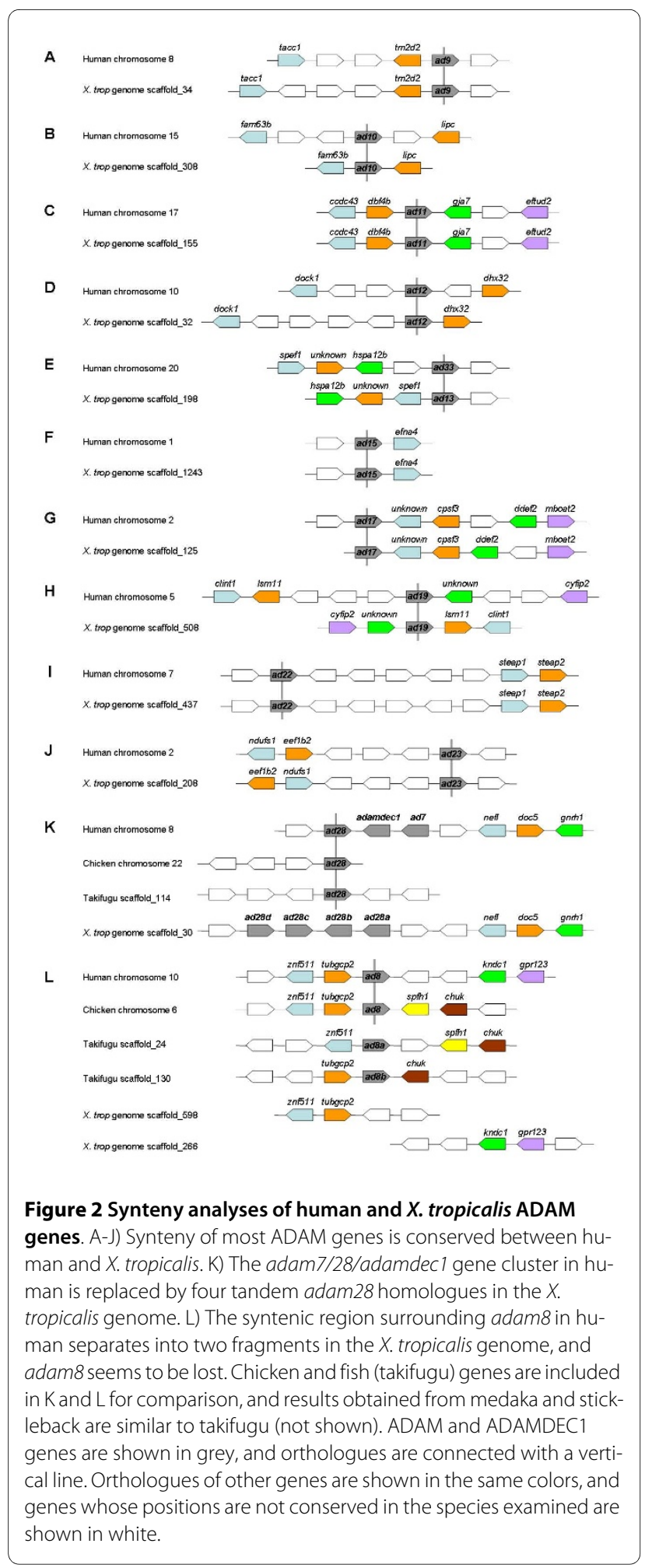

the loci of adam8, tubgcp 2 and $z n f 511$ are also conserved in the chicken genome, $k n d c 1$ and gpr123 genes are not localized in the vicinity; instead two other genes, spfh1 and chuk, are found in this region (Figure 2L). There are two adam 8 homologues in fish (takifugu), one flanked by znf511 and spfh1/chuk, and the other by tubgcp 2 and an additional homologue of chuk (Figure 2L), suggesting possible genome duplication associated with gene loss in this region. In the $X$. tropicalis genome, tubgcp 2 and $z n f 511$ were localized to scaffold_598, whereas $k n d c 1$ and gpr123 were localized to a different scaffold, scaffold_266 (Figure 2L), and spfh1 and chuk lie separately on two other scaffolds (not shown). No adam8 homologue was found on any of these scaffolds. These results suggest that this genomic region has undergone extensive rearrangement during evolution, and that the adam 8 gene was probably lost in the frogs. The possible absence of an ADAM8 orthologue in the $X$. tropicalis genome may reflect a poor conservation of this ADAM metalloproteinase during evolution. We also cannot rule out the possibility that the available genome sequence data may be incomplete.

In addition to potential loss of ADAM genes as compared to the mammalian genomes, there is also evidence for additional ADAM genes in the $X$. tropicalis genome. A homologue of ADAM10, which we named ADAM10like, was found in Xenopus but absent in most placental mammals. We also identified four homologues of ADAM28 on Scaffold_30 of the X. tropicalis genome assembly. Features of $X$. tropicalis ADAMs identified in this study are discussed in the following sections in terms of different ADAM clades as indicated in Figure 1A.

\section{ADAMs 12, 13 and 19}

We previously identified $X$. laevis ADAM13 as a proteolytically active ADAM expressed predominantly in cranial neural crest $(\mathrm{CNC})$ cells [44], an embryonic cell population that gives rise to craniofacial structures in vertebrates [55,56]. Ectopic expression of a protease-dead mutant of ADAM13, as well as antisense morpholino mediated knockdown of ADAM13, inhibits CNC migration in neurula stage embryos [34,40]. A closely related ADAM, ADAM19, was recently found to be required for $\mathrm{CNC}$ induction and migration in X. laevis [45]. The similarity between the in vivo functions of ADAMs 13 and 19 raises the possibility that these two ADAM metalloproteinases may act through similar mechanisms (e.g. by cleaving the same physiologically relevant substrates). Furthermore, there may be additional paralogues with similar roles in CNC development encoded by the frog genome. To address these questions we performed a search for closely related homologues of $X$. laevis ADAMs 13 and 19 in X. tropicalis.

Analysis of the $X$. tropicalis genome indicates that there are three ADAMs closely related to $X$. laevis ADAM13: $X$. tropicalis ADAMs 12, 13 and 19. We obtained full-length cDNA constructs for these ADAMs by direct cloning. As shown in Figure 3A, sequence comparison with other ADAM family members suggests that these three pro- 
teins constitute an ADAM subfamily with clear orthologous relationships between Xenopus and mammalian ADAMs 12 and 19. Although the relationship is not as clear, the most parsimonious explanation is that Xenopus ADAM13 and mammalian ADAM33 are orthologues. This is further supported by the synteny analysis, which shows a conserved genetic linkage between adam13/33 and spef1, hspa $12 b$ and an unknown gene (Figure 2E). ADAM12, 13/33, and 19 orthologs are detectable in zebrafish, suggesting that this ADAM subfamily was present in the ancestral vertebrate (Figure 3A). RT-PCR analyses showed that ADAMs 12, 13 and 19 are each expressed by neurula stage in $X$. tropicalis (Figure 3B; PCR products were confirmed by DNA sequencing). Functions of these ADAMs in CNC and neural develop-

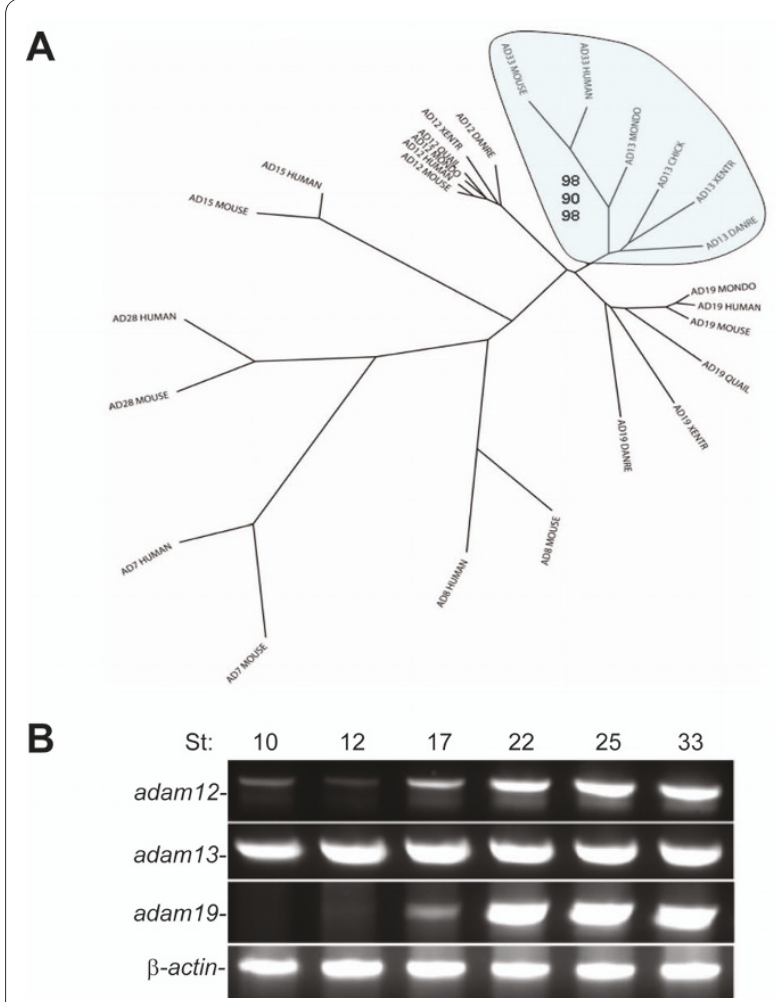

Figure 3 Sequence and expression analyses of ADAMs 12, 13/33, and 19. A) Phylogenetic analysis of selected ADAM family members including gene models from opossum and fish. A subset of the ADAM family from human, mouse, opossum (MONDO), bird (QUAIL, CHICK), $X$. tropicalis, and zebrafish (DANRE) was aligned and a neighbor-joining tree was drawn using ClustalX. The ADAM13/33 clade is highlighted, and numbers are bootstrap values (in percentage) from Neighbor (top), Protpars (middle), and Proml (bottom) analyses (see Additional File 6, D-F for trees generated using these models). B) Developmental expression of adams 12,13, and 19. RNA from X. tropicalis embryos at the indicated stages was analyzed for the presence of adam12, 13, and 19 transcripts by RT-PCR. Primer pairs for all adams were designed to span at least one large intron, so that the PCR products will not be contaminated by amplification of genomic DNA. PCR products for all 3 adams were confirmed by DNA sequencing. ment will be discussed separately (Wei et al., manuscript submitted).

\section{ADAM15}

Human ADAM15 was originally named metargidin (metalloprotease-RGD-disintegrin protein), because it was the first cellular disintegrin protein (and to date the only human ADAM) found to contain the consensus RGD integrin binding site in the disintegrin domain [57]. However, this RGD sequence is not conserved in mouse ADAM15 [58]. The mammalian ADAM15 gene encodes an active metalloproteinase, and several substrates have been identified for this enzyme [59-61]. Mice lacking ADAM15 do not show any apparent deficiencies, but they are more resistant to pathological neovascularization [62].

We searched the $X$. tropicalis genome and EST databases and identified an ADAM15 homologue that is 44\% identical to the human protein. Similar to human adam15 [46], the gene encoding this homologue was localized next to efna4 in the $X$. tropicalis genome (Figure $2 \mathrm{~F}$ ), suggesting that it is the orthologue of ADAM15. A cDNA clone encoding the $X$. laevis orthologue of this protein was also found in the EST databases (BC146626). Surprisingly, unlike the mammalian ADAM15 proteins, which contain the consensus zinc-binding motif HEXGH in the catalytic domain, both $X$. tropicalis ADAM15 and its $X$. laevis orthologue have the sequence HQXGH in this position (Figure 4). An E to Q mutation in the same motif has been shown to result in loss of proteolytic activity in ADAM12 [63], hence it is likely that frogs do not have an active ADAM15 metalloproteinase. Mammalian ADAM15 contains a proline-rich cytoplasmic tail with several potential Src homology-3 (SH3) domain binding sites [64]. As shown in Figure 4, many of these prolines are conserved in mammals and frogs. In contrast, while the mammalian ADAM15 proteins share a strikingly similar signal peptide, this peptide is less conserved in Xenopus ADAM15 (Additional File 2). Finally, primate, canine and bovine ADAM15 proteins have a consensus RGD integrin binding site in the disintegrin domain; this sequence is not conserved in rodent or frog ADAM15. Instead Xenopus ADAM15 proteins contain an RGD sequence within the cysteine-rich domain (Figure 4). Interestingly, this second RGD sequence is also present in canine and bovine ADAM15, whereas in the primate and rodent orthologues it is replaced by the sequence RGN (Figure 4). A possible explanation is that the ancestor of vertebrate ADAM15 might have two RGD integrin binding sites, one in the disintegrin domain and the other in the cysteine-rich domain. Both of these RGD sequences were maintained in the canine and bovine lineages (both belong to Laurasiatheria) but lost in rodents, while primates and frogs each retained a different RGD sequence. 


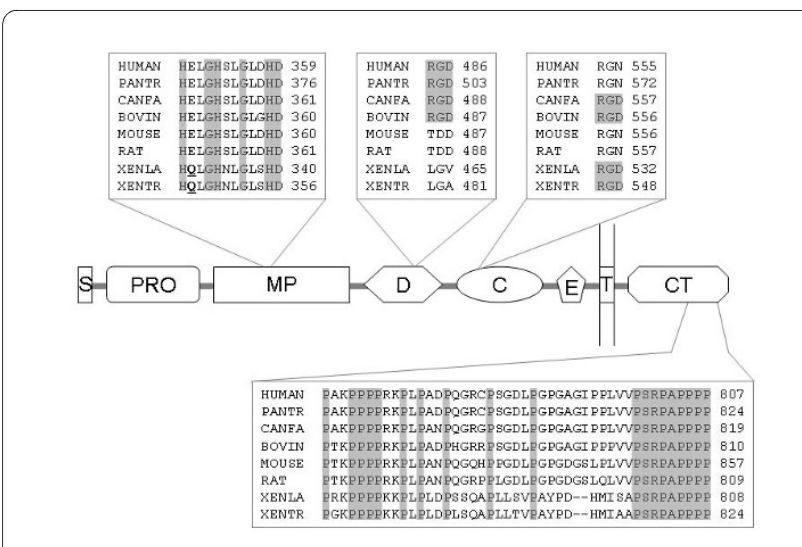

Figure 4 Sequence comparison of mammalian and Xenopus ADAM15. Sequence alignment of human, chimpanzee (PANTR), canine (CANFA), bovine (BOVIN), mouse, rat, X. laevis (XENLA) and X. tropicalis ADAM15 was generated using ClustalX. Domain organization of ADAM15 is shown with insets displaying alignments of the zinc-binding motif (the GIn in Xenopus ADAM15 is highlighted in bold and underscored), the RGD integrin-binding sites, and the conserved proline residues in the cytoplasmic tail. Conserved residues are highlighted in grey. See Figure 1 legend for abbreviations of domain names and Additional File 2 for a complete sequence alignment that also includes zebrafish ADAM15 proteins.

The conservation of the synteny, the SH3 binding motifs and the RGD sequences indicates that these Xenopus homologues are real orthologues of mammalian ADAM15, although the metalloproteinase consensus sequence was lost during evolution. In contrast, the two zebrafish ADAM15 homologues both contain the conserved zinc-binding motif but lack either RGD site (Additional File 2).

\section{ADAM28}

ADAM28 (also known as MDC-L or eMDC II) is a proteolytically active ADAM that is highly expressed in the epididymis and in lymphocytes [65-67]. Several alternatively spliced forms of ADAM28 have been detected in vivo, including a soluble form without a transmembrane region or cytoplasimc tail $[66,67]$. ADAM7, although proteolytically inactive, is closely related to ADAM28 (Figure 1). Genes encoding ADAM7, ADAM28, and ADAMDEC1 (Decysin) form a metalloproteinase gene cluster on human chromosome $8 \mathrm{p} 12$, presumably as a result of gene duplication [68]. ADAMDEC1 is a soluble ADAM-like protein lacking part of the disintegrin domain and the entire cycteine-rich domain; a conserved histidine residue in the zinc-binding motif is replaced by aspartate, but such a replacement was thought to have no negative effect on the metalloproteinase activity [68]. Expression of ADAMDEC1 is restricted to the immune system and is regulated by various stimuli during monocyte differentiation [69].

As discussed above, no Xenopus orthologue of ADAM7 was identified in this analysis. ADAMDEC1 seems to exist only in mammals [12], and we were unable to identify any likely orthologue in the $X$. tropicalis genome or in $X$. tropicalis/X. laevis EST databases. However, a BLAST search against the $X$. tropicalis genome assembly yielded four potential genes possibly encoding ADAM28 homologues on Scaffold_30 (Figure 2K). Although these potential genes have only slightly higher sequence similarities to ADAM28 than to ADAM7, the deduced protein sequences all contain a conserved zinc-binding motif (Additional File 3 and data not shown). Therefore they are considered homologues of adam28 (as ADAM7 is proteolytically inactive and seems to be restricted to mammals), and were assigned the names adams $28 a-d$. We obtained full-length cDNA clones for adams $28 a$ and $28 \mathrm{~b}$, and found two partial clones for adam $28 \mathrm{c}$ (CX934006 and CF377167). In addition, we identified a full-length cDNA encoding a soluble form of ADAM28b (ADAM28bs) in X. laevis EST databases (Additional File 3 ). The presence of soluble ADAM28 in both humans and frogs indicates a potentially conserved function of a diffusible ADAM28 metalloproteinase in vivo. A sequence alignment for frog and mammalian ADAM28 (including the soluble forms) is shown in Additional File 3. As in the case of human chromosome 8 , the cluster of adam 28 homologue genes on Scaffold_30 of the X. tropicalis genome may also represent gene duplication events in this region. Sequence comparison of the four $X$. tropicalis ADAM28 homologues with human ADAM7 and ADAMDEC1 nevertheless indicates no orthologous relationship between them (not shown). Therefore these duplication events in the frog and human genomes may have happened separately after the divergence of amphibians from mammals. Consistent with this hypothesis, both chicken and fish genomes lack such duplication in this region (Figure $2 \mathrm{~K}$ ).

\section{ADAM9}

ADAM9 (MDC9) was among the three active ADAM metalloproteinases (the other two were ADAMs 12 and 19) first identified in myoblasts. Because one of these ADAMs, ADAM12, is required for myotube formation in a myoblast cell line and in certain muscle tissues in vivo, these three ADAMs were also called the meltrins $[14,70]$. However, ADAM9 is not the most closely related paralogue of ADAMs 12 and 19 (Figure 1), and no apparent phenotype in muscle development was observed in adam 9-/- mice, although a recent report showed that they eventually develop retinal degeneration [47]. These findings suggest that ADAM9 does not belong to the meltrin clade.

An X. laevis orthologue of ADAM9 (xMDC9) was cloned in a previous study and, like its mouse orthologue, was found to be ubiquitously expressed at different developmental stages [43]. In this study we identified a fulllength EST clone encoding the putative $X$. tropicalis 
orthologue of ADAM9. Like their mammalian and zebrafish orthologues, both $X$. tropicalis and $X$. laevis ADAM9 proteins contain a proline-rich cytoplasmic tail (Figure 5 and Additional File 4). Two SH3 binding motifs have been identified previously in the cytoplasmic tails of human and mouse ADAM9, of which the C-terminal proximal one is also conserved in all the tetrapod species examined, including both X. tropicalis and X. laevis (Figure 5).

\section{ADAMs 11,22 and 23}

Three proteolytically inactive ADAMs, ADAMs 11, 22 and 23 (also known as MDC, MDC2 and MDC3, respectively), form an ADAM clade that is highly expressed in the brain [71]. Studies with knockout mice revealed that each of these ADAMs is required for normal neuronal function $[15,16,49,50]$. It was recently shown that ADAM22 functions as a receptor for the secreted protein LGI1. Mutations in LGI1 cause heritable epilepsies in humans, and one of these mutated forms of LGI1 fails to bind ADAM22 [29,72]. In a separate study, ADAMs 11, 22 and 23 were all shown to interact with LGI1 and the closely related LGI4 [28], suggesting that there may be a common mechanism for these ADAMs in maintaining normal brain function. Two $X$. laevis cDNA clones encoding different ADAMs, one partial (for xMDC11a) and one full-length (for xMDC11b), were described previously [43]. Our sequence comparison with the $X$. tropicalis and human genomes indicates that they are orthologues of mammalian ADAMs 11 and 22, respectively (not shown). X. laevis ADAM11 is highly expressed

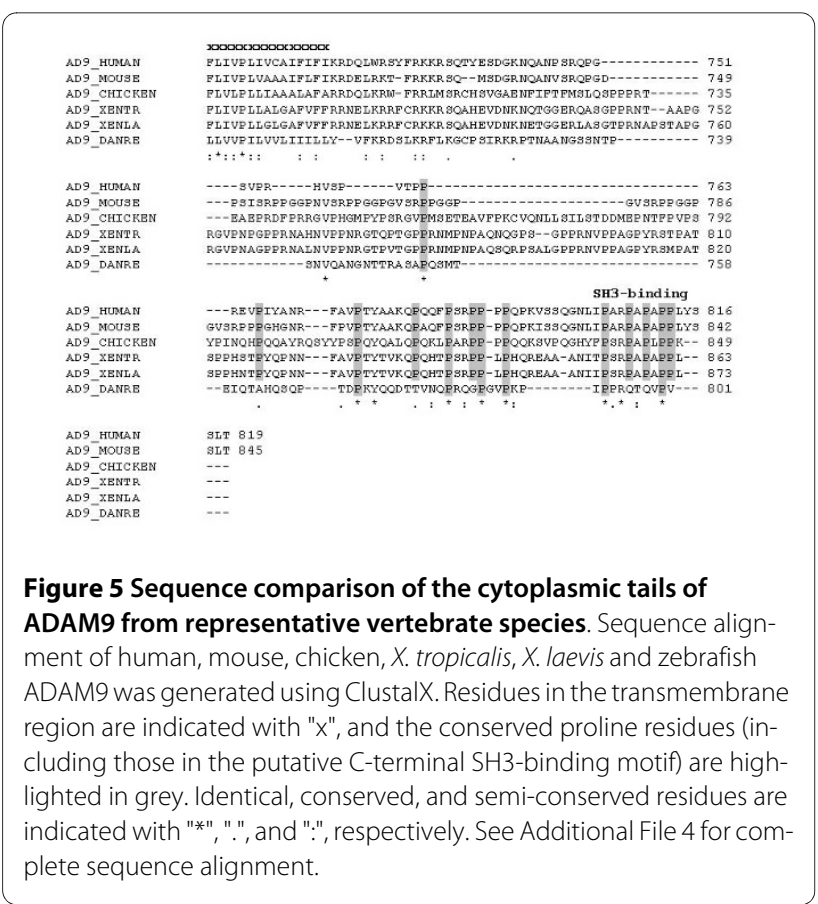

in CNC cells [43], but no functional study for this ADAM has been reported to date.

Here we report the full-length cDNA sequences for $X$. tropicalis ADAMs 11, 22 and 23. As shown in Table 1, these three $X$. tropicalis ADAMs resemble their human orthologues in both the deduced protein sequence and the high expression level in the brain [71]. ADAM11 is dispensable for survival in mice, but has important roles in spatial learning, motor coordination and nociceptive response $[15,16]$. Like its mammalian orthologues, $X$. tropicalis ADAM11 also has a very short cytoplasmic tail (Additional File 5). However, it lacks a large portion of the propeptide that is present in human, mouse and zebrafish ADAM11 (Figure 6A and Additional File 5). A survey of the exon-intron boundaries suggests that this difference arises from adoption of different splice sites, as exons 3,4 , 6, 7 and part of exon 5 in human adam11 (and mouse and zebrafish adam11; data not shown) are absent in the $X$. tropicalis adam11 transcript, while the other exons are conserved between these two species (Figures. 1B and $6 \mathrm{~B})$. Because ADAM11 does not have the zinc-binding motif that is required for metalloproteinase activity, it is conceivable that part of the ADAM11 propeptide may have been lost in frogs during evolution.

Mice lacking either ADAM22 or 23 develop severe ataxia and die shortly after birth, and the similarity in phenotype has led to the speculation that these two ADAMs may act together in a nonredundant way $[49,50]$. Because of the similar abilities of ADAMs 11, 22 and 23 to interact with LGIs, it was also proposed that distinct functions of these ADAMs may depend on their expression in different cell types [28]. Interestingly, both human and mouse adam 22 transcripts have multiple alternatively spliced forms, and mouse cDNA libraries obtained from different types of cells contain different splice variants of adam22 [49,73]. We identified three differently spliced isoforms of ADAM22 in X. tropicalis, and sequence comparisons revealed that the previously published X. laevis ADAM22 (xMDC11b; O42596) represents yet another isoform (Figure 7A). In mammals, alternative splicing results in multiple transmembrane ADAM22 variants with different cytoplasmic tails; however, no soluble form has been reported to date [49,73]. Among the four Xenopus ADAM22 isoforms identified in this study, three (ISOs 1, 2 and 4) are transmembrane forms that differ in their cytoplasmic tails. Interestingly, the other isoform (ISO3) is a truncated variant that lacks a transmembrane region and a cytoplasmic tail (Figure 7B). Given the potential physiological role of ADAM22 as a cell surface receptor, this soluble form may provide a possible regulatory mechanism to control the functions of the transmembrane forms of ADAM22. It will be of interest to determine if the splicing of adam $22 \mathrm{mRNA}$ in Xenopus is also cell-type specific. 


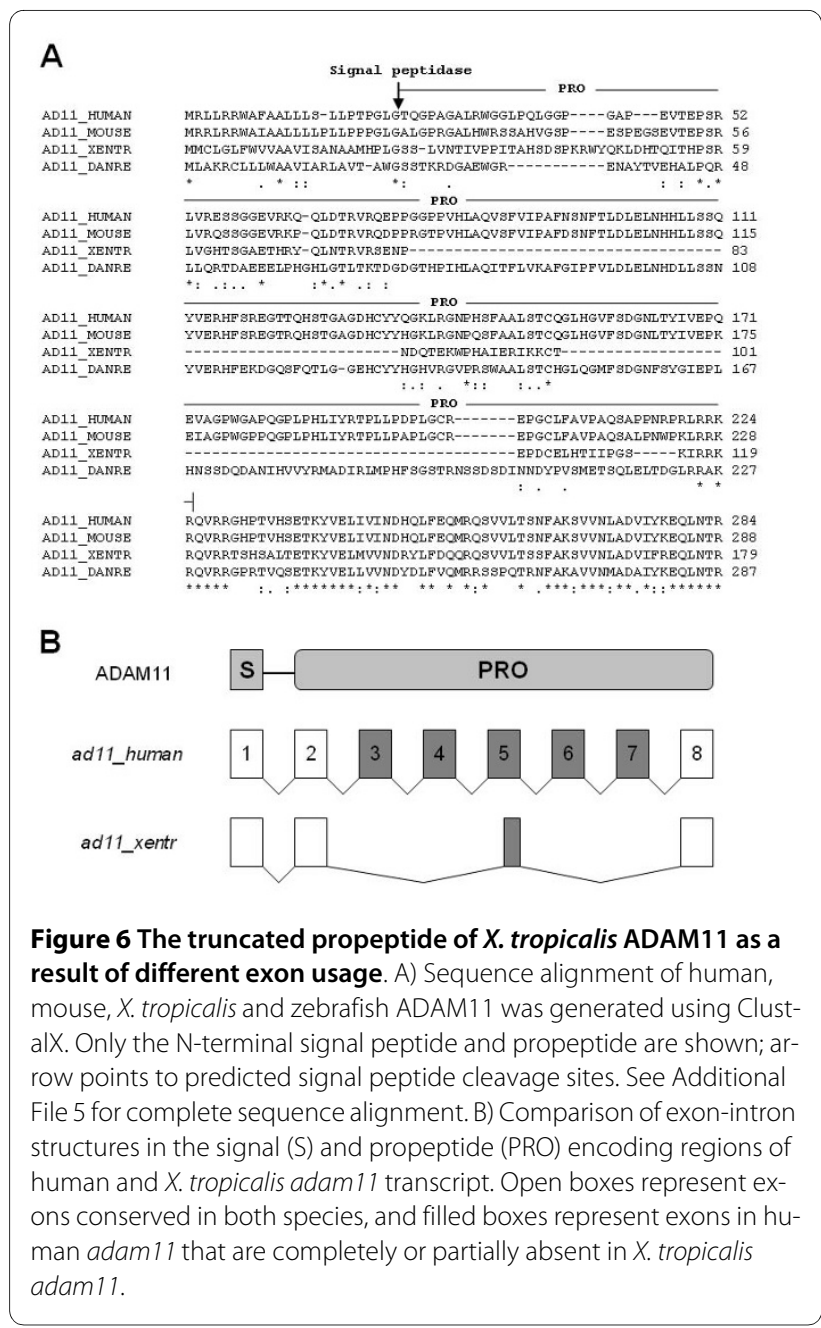

ADAMs 10, 10-like and 17

ADAMs 10 and 17 are probably the most extensively studied ADAMs because of their important roles in development and disease. ADAM10 (also known as Kuzbanian or KUZ) was originally discovered in Drosophila as an essential component of the Notch signaling pathway [74], while ADAM17 (also known as tumor necrosis factor- $\alpha$ converting enzyme or TACE) was first cloned in mice as the major enzyme required for shedding of the cytokine TNF- $\alpha$ [75,76]. ADAMs 10 and 17 share many substrates, and it is still a subject of debate as to which of these two enzymes is required for cleaving Notch receptors $[77,78]$. The Xenopus system has long been used to study Notch signaling, and ectopic expression of a dominant-negative form of mouse ADAM10 was shown to cause a Notch phenotype in X. laevis embryos [26]. Therefore it is important to identify the Xenopus homologues of ADAMs 10 and 17 and understand their respective roles in early development.

We identified cDNA clones for both $X$. tropicalis and $X$. laevis ADAM17 from Xenopus EST collections using

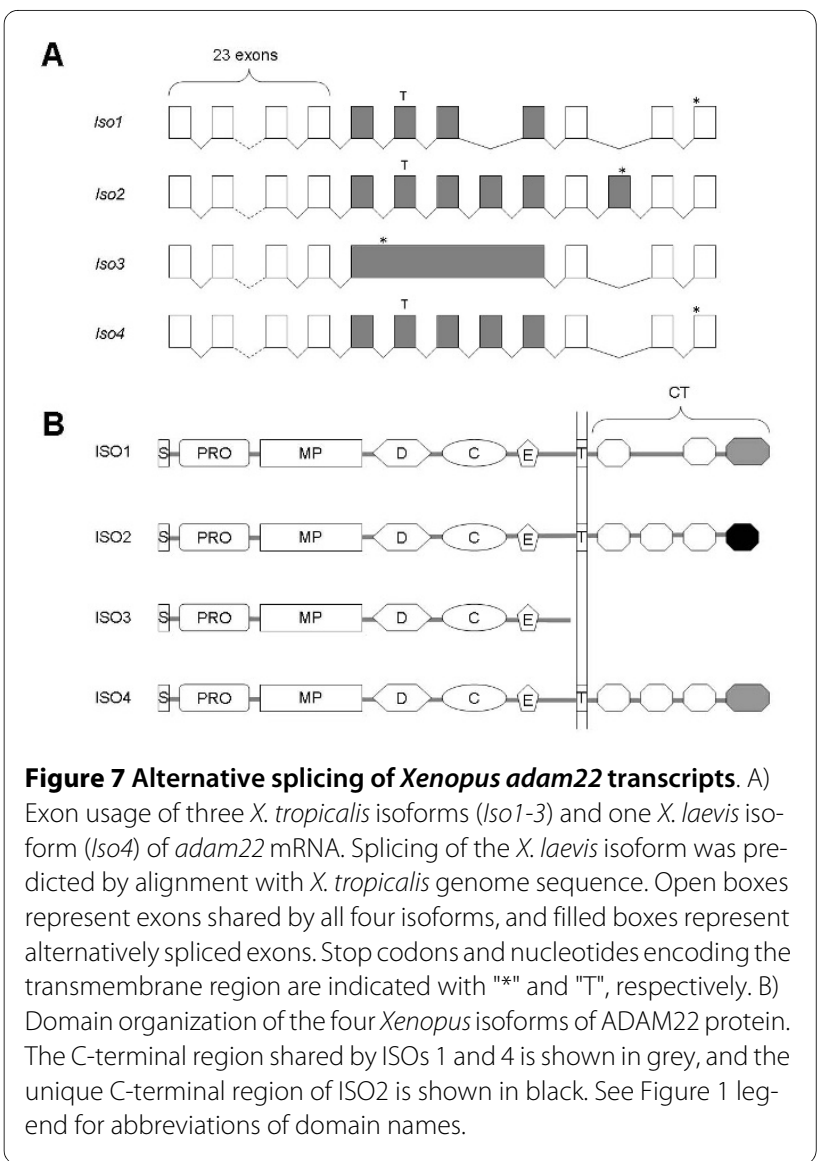

BLAST searches. Although it is not clear if the $X$. tropicalis genome contains a true TNF- $\alpha$ gene, these Xenopus ADAM17 proteases may still be involved in cleavage of other members of the TNF superfamily and/or ligands of the epidermal growth factor receptor, as their mammalian orthologues do [79]. In addition to our previous identification of $X$. laevis ADAM10 [7], we obtained a fulllength clone encoding $X$. tropicalis ADAM10. A pair-wise sequence comparison shows $82 \%$ identity between $X$. tropicalis and human ADAM10, the highest among all ADAMs identified in this study (Table 1), indicating that functions of this enzyme are likely conserved in frogs. In the Drosophila genome there exist two ADAM10 homologues, Kuzbanian (KUZ) and Kuz-like (KUL), both of which are required for Notch signaling in certain cells and tissues $[74,80]$. We also found an actively transcribed paralogue of ADAM10 in X. tropicalis, which we named ADAM10-like (Table 1). However, sequence comparisons suggest that ADAM10-like is not the orthologue of Drosophila KUL (Figure 8). Therefore these two ADAM10 paralogues in different species may have been derived by two independent gene duplication events. Homologues of ADAM10-like were found in some other animals, including $X$. laevis, zebrafish and a few mammalian species. Detailed sequence analysis and functional studies of 
ADAMs 10, 10-like and 17 will be reported elsewhere (Xu et al., manuscript in preparation). A phylogenetic tree estimating the evolution of ADAMs 10 and 17 (as well as KUL and X. tropicalis ADAM10-like) is shown in Figure 8.

\section{Conclusions}

ADAMs are widely involved in developmental and pathological processes through their activities as proteases and binding partners for other proteins. The recent emergence of Xenopus tropicalis as a powerful new vertebrate model for developmental and genetic studies prompted us to take a genome-wide approach to identify ADAM genes existing in this species. Using bioinformatics tools, we were able to obtain full-length sequence information for putative $X$. tropicalis orthologues of most mammalian non-testis specific ADAMs. The orthology between $X$. tropicalis and human ADAMs were further supported by conserved splicing patterns and synteny. The high sequence similarities between mammalian and Хenopus ADAMs 9, 10, 11, 12, 17, 19, 22 and 23 are in good agreement with their essential roles in vivo, as demonstrated by previous studies with knockout mice. Our sequence analyses also revealed the conservation of certain features, such as alternative splicing and cytoplasmic SH3 binding

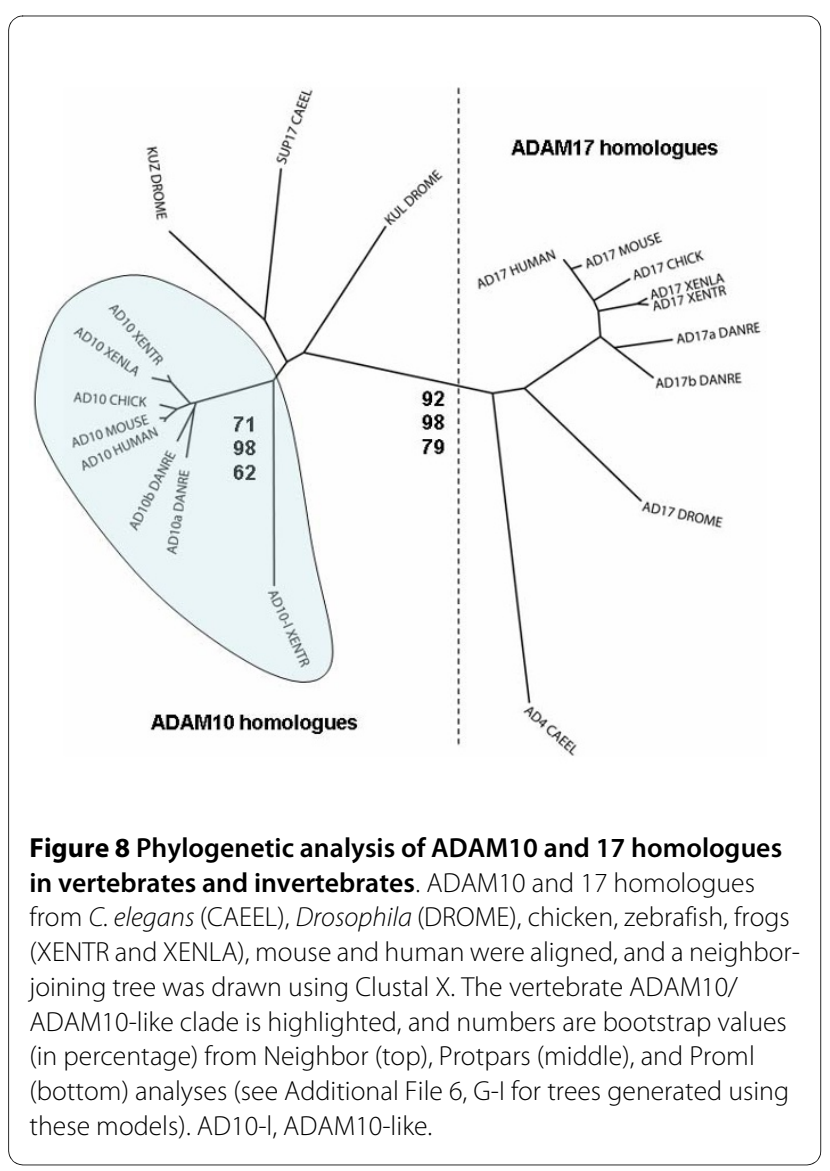

motifs, in some ADAMs. Functional characterization of these ADAMs (and specific motifs) in X. tropicalis may provide useful information to guide future research on human development and diseases. Evolutionary divergence is also evident by the potential loss of some ADAM genes and ADAM domains/motifs in Xenopus. Orthologues of ADAMs 7 and 8 were not detected in the current version of $X$. tropicalis genome sequence, and ADAM15 protease activity and part of the ADAM11 propeptide seem to be absent in $X$. tropicalis. These findings suggest that functions of these ADAMs or ADAM domains are likely not conserved. In contrast, the additional ADAM10- and ADAM28-like metalloproteinases, which presumably arose as results of gene duplication, may have conferred selective advantages during the evolution of amphibians. The sequence information described in this study provides the basis for understanding the evolution of ADAM functions in vertebrates.

\section{Methods \\ Bioinformatics}

Homologues of known human or X. laevis ADAM genes were identified by using sequence-based searches of the Joint Genome Institute (JGI) X. tropicalis genome assembly v4.1 [81], and cDNA constructs were obtained from IMAGE Consortium distributor (OpenBiosystems) or by experimental cloning as described below. All new $X$. tropicalis ADAM cDNAs reported here have been sequenced by the Biomolecular Research Facility at the University of Virginia, and assembled to give the final full-length sequences. Multiple sequence alignments were constructed with ClustalX 2.0 [82]. Phylogenetic trees were generated using ClustalX (neighbor joining), Phylip neighbor, Phylip protpars (maximum parsimony) and Phylip proml (maximum likelyhood) [83]. Trees were displayed using the Phylip application Drawtree. Because phylogenetic trees generated using different methods are consistent with each other, only those generated with ClustalX are shown in the main figures (see Additional File 6 for trees generated using the other models). For bootstrap analyses, bootstrap replicate groups were created using Phylip application Seqboot, and bootstrap values were calculated using Phylip Neighbor, Protpars, and Proml analyses (bootstrap values calculated with ClustalX are very similar to those calculated with Phylip Neighbor, therefore only the latter are shown). Synteny analyses were carried out using Metazome [84]. Exonintron structures were estimated by comparing the cDNA sequences with $X$. tropicalis genome sequence using BLAT searching from the UCSC Genome Bioinformatics Site [85]. Signal peptidase cleavage sites were assigned according to available sequence annotations (for known ADAMs) or predictions by SignalP (for new ADAMs identified in this study) [86]. Domain structure predic- 
tions were carried out using the Simple Modular Architecture Research Tool (SMART) [87].

The following human ADAM protein sequences were used to identify their $X$. tropicalis homologues: ADAM9 (Q13443), ADAM10 (O14672), ADAM11 (O75078), ADAM12 (O43184), ADAM15 (Q13444), ADAM17 (P78536), ADAM19 (Q9H013), ADAM22 (Q9P0K1), ADAM23 (O75077), ADAM28 (Q9UKQ2), and ADAM33 (Q9BZ11). Previously reported X. laevis ADAMs used in this study are: ADAM9/xMDC9 (NP_001079073), ADAM10 (Q8JIY1), ADAM11/ xMDC11a (Q9PSZ3), ADAM13 (AAI69959), ADAM19 (ACE82293), and ADAM22/xMDC11b (O42596). IMAGE clone IDs for ESTs encoding $X$. tropicalis and $X$. laevis ADAMs identified in this study are: $X$. tropicalis ADAM9 (7644280), ADAM10 (7644709), ADAM11 (7650432), ADAM15 (7693815), ADAM17 (6994025), ADAM22 isoform 1 (7658598), ADAM22 isoform 2 (8916700), ADAM22 isoform 3 (7650959), ADAM23 (7649052), ADAM28a (7716115), and ADAM28b (8898681); X. laevis ADAM15 (7978429; GenBank accession \# BC146626), ADAM17 (3199617; GenBank accession \# DQ287907), and ADAM28bs (5513111; GenBank accession \# $\underline{\mathrm{BC091726}}$.

\section{Cloning and RT-PCR}

Total RNA from $X$. tropicalis embryos at desired stages was prepared as described [88]. X. tropicalis orthologues of X. laevis adam 13 and mammalian adams 12 and 19, and $X$. tropicalis adam10-like gene were identified by searching against the $X$. tropicalis genome assembly, and the predicted sequences were used to design primers for RT-PCR to obtain partial clones. 5'- and 3'- RACE was then performed using BD Smart RACE kit (BD Biosciences), and full-length cDNA sequences were assembled into pCR2.1. RT-PCR experiments for expression of adams 12, 13, and 19 were carried out using total RNA purified from $X$. tropicalis embryos at different developmental stages. Primer sequences are shown in Additional File 7.

\section{Additional material}

Additional file 1 Phylogenetic tree of ADAMs from representative vertebrate species.

Additional file 2 Complete sequence alignment of ADAM15 from representative vertebrate species.

Additional file 3 Complete Sequence alignment of ADAM28 from representative vertebrate species.

Additional file 4 Complete sequence alignment of ADAM9 from representative vertebrate species.

Additional file 5 Complete sequence alignment of ADAM11 from representative vertebrate species.

Additional file 6 Phylogenetic trees generated using alternative models.

Additional file 7 Sequences of primers used in RT-PCR experiments shown in Figure 3B.
Authors' contributions

SW conceived of the study, carried out most of the experiments and data analyses, and drafted the manuscript. CAW performed the sequence alignment and phylogenetic analyses, and helped draft the manuscript. GX, LCB and AS participated in the cloning and RT-PCR experiments. DWD and JMW participated in the design and coordination of this study, and helped draft the manuscript. All authors read and approved the final manuscript.

\section{Acknowledgements}

This work was supported by the National Institute of Health (DE14365 and HD26402 to DWD) and March of Dimes Foundation (grant F405-140 to JMW and 1-FY10-399 to DWD and SW). SW was supported by an American Heart Association postdoctoral fellowship, and LCB was supported by a training grant to the University of Virginia (5T32GA09109) and by a Ruth L. Kirschstein postdoctoral fellowship.

\section{Author Details}

1Department of Cell Biology and the Morphogenesis and Regenerative Medicine Institute, University of Virginia, Charlottesville, VA 22908, USA, 2David $\mathrm{H}$. Koch Institute for Integrative Cancer Research at Massachusetts Institute of Technology, Cambridge, MA 02139, USA and 3Department of Chemistry, University of Central Arkansas, Conway, AR 72035, USA

Received: 4 February 2010 Accepted: 14 July 2010

Published: 14 July 2010

\section{References}

1. Rawlings ND, Morton FR, Kok CY, Kong J, Barrett AJ: MEROPS: the peptidase database. Nucleic Acids Res 2008, 36:D320-325.

2. White JM, Bridges LC, DeSimone DW, Tomczuk M, Wolfsberg TG: Introduction to the ADAM family. In The ADAM Family of Proteases Edited by: Hooper NM, Lendeckel U. Dordrecht, Springer; 2005:1-28.

3. Blobel CP: ADAMs: key components in EGFR signalling and development. Nat Rev Mol Cell Biol 2005, 6:32-43.

4. Huang J, Bridges LC, White JM: Selective modulation of integrinmediated cell migration by distinct ADAM family members. Mol Biol Cell 2005, 16:4982-4991.

5. Gaultier A, Cousin H, Darribere T, Alfandari D: ADAM13 disintegrin and cysteine-rich domains bind to the second heparin-binding domain of fibronectin. J Biol Chem 2002, 277:23336-23344.

6. Thodeti CK, Albrechtsen R, Grauslund M, Asmar M, Larsson C, Takada Y Mercurio AM, Couchman JR, Wewer UM: ADAM12/syndecan-4 signaling promotes beta 1 integrin-dependent cell spreading through protein kinase Calpha and RhoA. J Biol Chem 2003, 278:9576-9584

7. Smith KM, Gaultier A, Cousin H, Alfandari D, White JM, DeSimone DW: The cysteine-rich domain regulates ADAM protease function in vivo. $J$ Cell Biol 2002, 159:893-902.

8. Janes PW, Saha N, Barton WA, Kolev MV, Wimmer-Kleikamp SH, Nievergall E, Blobel CP, Himanen JP, Lackmann M, Nikolov DB: Adam meets Eph: an ADAM substrate recognition module acts as a molecular switch for ephrin cleavage in trans. Cell 2005, 123:291-304.

9. Cousin H, Gaultier A, Bleux C, Darribere T, Alfandari D: PACSIN2 is a regulator of the metalloprotease/disintegrin ADAM13. Dev Bio/ 2000, 227:197-210.

10. Zheng Y, Schlondorff J, Blobel CP: Evidence for regulation of the tumor necrosis factor alpha-convertase (TACE) by protein-tyrosine phosphatase PTPH1. J Biol Chem 2002, 277:42463-42470

11. Tanaka M, Nanba D, Mori S, Shiba F, Ishiguro H, Yoshino K, Matsuura N, Higashiyama S: ADAM binding protein Eve-1 is required for ectodomain shedding of epidermal growth factor receptor ligands. J Biol Chem 2004, 279:41950-41959.

12. ADAM family tree in Treefam [http://www.treefam.org/cgi-bin/ treeview.pl?ac=TF314733\&stype $=$ full]

13. Cho C: Mammalian ADAMs with testis-specific or -predominant expression. In The ADAM Family of Proteases Edited by: Hooper NM, Lendeckel U. Dordrecht, Springer; 2005:239-259.

14. Kurisaki T, Masuda A, Sudo K, Sakagami J, Higashiyama S, Matsuda Y, Nagabukuro A, Tsuji A, Nabeshima Y, Asano M, et al:: Phenotypic analysis of Meltrin alpha (ADAM12)-deficient mice: involvement of Meltrin alpha in adipogenesis and myogenesis. Mol Cell Biol 2003, 23:55-61.

15. Takahashi E, Sagane K, Oki T, Yamazaki K, Nagasu T, Kuromitsu J: Deficits in spatial learning and motor coordination in ADAM11-deficient mice. BMC Neurosci 2006, 7:19. 
16. Takahashi E, Sagane K, Nagasu T, Kuromitsu J: Altered nociceptive response in ADAM11-deficient mice. Brain Res 2006, 1097:39-42.

17. Hartmann D, de Strooper B, Serneels L, Craessaerts K, Herreman A, Annaert W, Umans L, Lubke T, Lena Illert A, von Figura K, et al.: The disintegrin/metalloprotease ADAM 10 is essential for Notch signalling but not for alpha-secretase activity in fibroblasts. Hum Mol Genet 2002, 11:2615-2624

18. Peschon JJ, Slack JL, Reddy P, Stocking KL, Sunnarborg SW, Lee DC, Russell WE, Castner BJ, Johnson RS, Fitzner JN, et al:: An essential role for ectodomain shedding in mammalian development. Science 1998, 282:1281-1284

19. Zhou HM, Weskamp G, Chesneau V, Sahin U, Vortkamp A, Horiuchi K, Chiusaroli R, Hahn R, Wilkes D, Fisher P, et al:: Essential role for ADAM19 in cardiovascular morphogenesis. Mol Cell Biol 2004, 24:96-104.

20. Huovila AP, Turner AJ, Pelto-Huikko M, Karkkainen I, Ortiz RM: Shedding light on ADAM metalloproteinases. Trends Biochem Sci 2005, 30:413-422

21. Asakura M, Kitakaze M, Takashima S, Liao Y, Ishikura F, Yoshinaka T, Ohmoto H, Node K, Yoshino K, Ishiguro H, et al:: Cardiac hypertrophy is inhibited by antagonism of ADAM12 processing of HB-EGF: metalloproteinase inhibitors as a new therapy. Nat Med 2002, 8:35-40.

22. Black RA: Tumor necrosis factor-alpha converting enzyme. Int J Biochem Cell Biol 2002, 34:1-5.

23. Overall CM, Blobel CP: In search of partners: linking extracellular proteases to substrates. Nat Rev Mol Cell Biol 2007, 8:245-257.

24. Jarriault $S$, Greenwald I: Evidence for functional redundancy between $C$. elegans ADAM proteins SUP-17/Kuzbanian and ADM-4/TACE. Dev Biol 2005, 287:1-10

25. Horiuchi K, Zhou HM, Kelly K, Manova K, Blobel CP: Evaluation of the contributions of ADAMs $9,12,15,17$, and 19 to heart development and ectodomain shedding of neuregulins beta1 and beta2. Dev Biol 2005 283:459-471.

26. Pan D, Rubin GM: Kuzbanian controls proteolytic processing of Notch and mediates lateral inhibition during Drosophila and vertebrate neurogenesis. Cell 1997, 90:271-280.

27. Sardi SP, Murtie J, Koirala S, Patten BA, Corfas G: Presenilin-dependent ErbB4 nuclear signaling regulates the timing of astrogenesis in the developing brain. Cell 2006, 127:185-197.

28. Sagane K, Ishihama Y, Sugimoto H: LGI1 and LGI4 bind to ADAM22, ADAM23 and ADAM11. Int J Biol Sci 2008, 4:387-396.

29. Fukata Y, Adesnik H, Iwanaga T, Bredt DS, Nicoll RA, Fukata M: Epilepsyrelated ligand/receptor complex LGI1 and ADAM22 regulate synaptic transmission. Science 2006, 313:1792-1795.

30. Edwards DR, Handsley MM, Pennington CJ: The ADAM metalloproteinases. Mol Aspects Med 2008, 29:258-289.

31. Reiss K, Ludwig A, Saftig P: Breaking up the tie: disintegrin-like metalloproteinases as regulators of cell migration in inflammation and invasion. Pharmacol Ther 2006, 111:985-1006.

32. Iba K, Albrechtsen R, Gilpin B, Frohlich C, Loechel F, Zolkiewska A, Ishiguro K, Kojima T, Liu W, Langford JK, et al.: The cysteine-rich domain of human ADAM 12 supports cell adhesion through syndecans and triggers signaling events that lead to beta 1 integrin-dependent cell spreading. J Cell Biol 2000, 149:1143-1156.

33. Millichip MI, Dallas DJ, Wu E, Dale S, McKie N: The metallo-disintegrin ADAM10 (MADM) from bovine kidney has type IV collagenase activity in vitro. Biochem Biophys Res Commun 1998, 245:594-598.

34. Alfandari D, Cousin H, Gaultier A, Smith K, White JM, Darribere T, DeSimone DW: Xenopus ADAM 13 is a metalloprotease required for cranial neural crest-cell migration. Curr Biol 2001, 11:918-930.

35. Roy R, Wewer UM, Zurakowski D, Pories SE, Moses MA: ADAM 12 cleaves extracellular matrix proteins and correlates with cancer status and stage. J Biol Chem 2004, 279:51323-51330.

36. Maretzky T, Reiss K, Ludwig A, Buchholz J, Scholz F, Proksch E, de Strooper B, Hartmann D, Saftig P: ADAM10 mediates E-cadherin shedding and regulates epithelial cell-cell adhesion, migration, and beta-catenin translocation. Proc Natl Acad Sci USA 2005, 102:9182-9187.

37. Reiss K, Maretzky T, Ludwig A, Tousseyn T, de Strooper B, Hartmann D, Saftig P: ADAM10 cleavage of $\mathrm{N}$-cadherin and regulation of cell-cell adhesion and beta-catenin nuclear signalling. EMBO J 2005, 24:742-752

38. Reiss K, Maretzky T, Haas IG, Schulte M, Ludwig A, Frank M, Saftig P. Regulated ADAM10-dependent ectodomain shedding of gamma- protocadherin C3 modulates cell-cell adhesion. J Biol Chem 2006, 281:21735-21744

39. Bech-Serra JJ, Santiago-Josefat B, Esselens C, Saftig P, Baselga J, Arribas J, Canals F: Proteomic identification of desmoglein 2 and activated leukocyte cell adhesion molecule as substrates of ADAM17 and ADAM 10 by difference gel electrophoresis. Mol Cell Biol 2006, 26:5086-5095.

40. McCusker C, Cousin H, Neuner R, Alfandari D: Extracellular cleavage of cadherin- 11 by ADAM metalloproteases is essential for Xenopus cranial neural crest cell migration. Mol Biol Cell 2009, 20:78-89.

41. Fu L, Das B, Mathew S, Shi YB: Genome-wide identification of Xenopus matrix metalloproteinases: conservation and unique duplications in amphibians. BMC Genomics 2009, 10:81.

42. Shilling FM, Kratzschmar J, Cai H, Weskamp G, Gayko U, Leibow J, Myles DG, Nuccitelli R, Blobel CP: Identification of metalloprotease/ disintegrins in Xenopus laevis testis with a potential role in fertilization. Dev Biol 1997, 186:155-164.

43. Cai H, Kratzschmar J, Alfandari D, Hunnicutt G, Blobel CP: Neural crestspecific and general expression of distinct metalloproteasedisintegrins in early Xenopus laevis development. Dev Biol 1998, 204:508-524.

44. Alfandari D, Wolfsberg TG, White JM, DeSimone DW: ADAM 13: a novel ADAM expressed in somitic mesoderm and neural crest cells during Xenopus laevis development. Dev Biol 1997, 182:314-330.

45. Neuner R, Cousin H, McCusker C, Coyne M, Alfandari D: Xenopus ADAM19 is involved in neural, neural crest and muscle development. Mech Dev 2009, 126:240-255.

46. Kleino I, Ortiz RM, Huovila AP: ADAM15 gene structure and differential alternative exon use in human tissues. BMC Mol Biol 2007, 8:90.

47. Parry DA, Toomes C, Bida L, Danciger M, Towns KV, McKibbin M, Jacobson SG, Logan CV, Ali M, Bond J, et al:: Loss of the metalloprotease ADAM9 leads to cone-rod dystrophy in humans and retinal degeneration in mice. Am J Hum Genet 2009, 84:683-691.

48. Kurohara K, Komatsu K, Kurisaki T, Masuda A, Irie N, Asano M, Sudo K, Nabeshima $Y$, Iwakura $Y$, Sehara-Fujisawa A: Essential roles of Meltrin beta (ADAM19) in heart development. Dev Biol 2004, 267:14-28.

49. Sagane K, Hayakawa K, Kai J, Hirohashi T, Takahashi E, Miyamoto N, Ino M, Oki T, Yamazaki K, Nagasu T: Ataxia and peripheral nerve hypomyelination in ADAM22-deficient mice. BMC Neurosci 2005, 6:33.

50. Mitchell KJ, Pinson KI, Kelly OG, Brennan J, Zupicich J, Scherz P, Leighton PA, Goodrich LV, Lu X, Avery BJ, et al.: Functional analysis of secreted and transmembrane proteins critical to mouse development. Nat Genet 2001, 28:241-249.

51. Cornwall GA, Hsia N: ADAM7, a member of the ADAM (a disintegrin and metalloprotease) gene family is specifically expressed in the mouse anterior pituitary and epididymis. Endocrinology 1997, 138:4262-4272.

52. UniGene [http://www.ncbi.nlm.nih.gov/unigene]

53. Koller G, Schlomann U, Golfi P, Ferdous T, Naus S, Bartsch JW: ADAM8/ MS2/CD156, an emerging drug target in the treatment of inflammatory and invasive pathologies. Curr Pharm Des 2009, 15:2272-2281

54. Kelly K, Hutchinson G, Nebenius-Oosthuizen D, Smith AJ, Bartsch JW, Horiuchi K, Rittger A, Manova K, Docherty AJ, Blobel CP: Metalloproteasedisintegrin ADAM8: expression analysis and targeted deletion in mice. Dev Dyn 2005, 232:221-231.

55. Trainor $P$, Nieto MA: Jawsfest: new perspectives on neural crest lineages and morphogenesis. Development 2003, 130:5059-5063.

56. Helms JA, Schneider RA: Cranial skeletal biology. Nature 2003, 423:326-331.

57. Kratzschmar J, Lum L, Blobel CP: Metargidin, a membrane-anchored metalloprotease-disintegrin protein with an RGD integrin binding sequence. J Biol Chem 1996, 271:4593-4596.

58. Lum L, Reid MS, Blobel CP: Intracellular maturation of the mouse metalloprotease disintegrin MDC15. J Biol Chem 1998, 273:26236-26247.

59. Maretzky T, Yang G, Ouerfelli O, Overall CM, Worpenberg S, Hassiepen U, Eder J, Blobel CP: Characterization of the catalytic activity of the membrane-anchored metalloproteinase ADAM15 in cell-based assays. Biochem J 2009, 420:105-113.

60. Najy AJ, Day KC, Day ML: The ectodomain shedding of E-cadherin by ADAM15 supports ErbB receptor activation. J Biol Chem 2008, 283:18393-18401 
61. Fourie AM, Coles F, Moreno V, Karlsson L: Catalytic activity of ADAM8, ADAM15, and MDC-L (ADAM28) on synthetic peptide substrates and in ectodomain cleavage of CD23. J Biol Chem 2003, 278:30469-30477.

62. Horiuchi K, Weskamp G, Lum L, Hammes HP, Cai H, Brodie TA, Ludwig T, Chiusaroli R, Baron R, Preissner KT, et al:: Potential role for ADAM15 in pathological neovascularization in mice. Mol Cell Biol 2003, 23:5614-5624

63. Loechel F, Gilpin BJ, Engvall E, Albrechtsen R, Wewer UM: Human ADAM 12 (meltrin alpha) is an active metalloprotease. J Biol Chem 1998, 273:16993-16997.

64. Kleino I, Ortiz RM, Yritys M, Huovila AP, Saksela K: Alternative splicing of ADAM15 regulates its interactions with cellular SH3 proteins. J Cell Biochem 2009, 108:877-885.

65. Jury JA, Perry AC, Hall L: Identification, sequence analysis and expression of transcripts encoding a putative metalloproteinase, eMDC II, in human and macaque epididymis. Mol Hum Reprod 1999 5:1127-1134.

66. Howard L, Maciewicz RA, Blobel CP: Cloning and characterization of ADAM28: evidence for autocatalytic pro-domain removal and for cell surface localization of mature ADAM28. Biochem J 2000, 348:21-27.

67. Roberts CM, Tani PH, Bridges LC, Laszik Z, Bowditch RD: MDC-L, a novel metalloprotease disintegrin cysteine-rich protein family member expressed by human lymphocytes. J Biol Chem 1999, 274:29251-29259.

68. Bates EE, Fridman WH, Mueller CG: The ADAMDEC1 (decysin) gene structure: evolution by duplication in a metalloprotease gene cluster on chromosome 8p12. Immunogenetics 2002, 54:96-105.

69. Fritsche J, Muller A, Hausmann M, Rogler G, Andreesen R, Kreutz M Inverse regulation of the ADAM-family members, decysin and MADDAM/ADAM19 during monocyte differentiation. Immunology 2003, 110:450-457.

70. Yagami-Hiromasa T, Sato T, Kurisaki T, Kamijo K, Nabeshima Y, FujisawaSehara A: A metalloprotease-disintegrin participating in myoblast fusion. Nature 1995, 377:652-656

71. Sagane K, Ohya Y, Hasegawa Y, Tanaka I: Metalloproteinase-like, disintegrin-like, cysteine-rich proteins MDC2 and MDC3: novel human cellular disintegrins highly expressed in the brain. Biochem J 1998, 334:93-98.

72. Kalachikov S, Evgrafov $O$, Ross B, Winawer M, Barker-Cummings C, Martinelli Boneschi F, Choi C, Morozov P, Das K, Teplitskaya E, et al: Mutations in LGI1 cause autosomal-dominant partial epilepsy with auditory features. Nature Genet 2002, 30:335-341.

73. Godde NJ, D'Abaco GM, Paradiso L, Novak U: Differential coding potential of ADAM22 mRNAs. Gene 2007, 403:80-88.

74. Rooke J, Pan D, Xu T, Rubin GM: KUZ, a conserved metalloproteasedisintegrin protein with two roles in Drosophila neurogenesis. Science 1996, 273:1227-1231.

75. Black RA, Rauch CT, Kozlosky CJ, Peschon JJ, Slack JL, Wolfson MF, Castner BJ, Stocking KL, Reddy P, Srinivasan S, et al:: A metalloproteinase disintegrin that releases tumour-necrosis factor-alpha from cells. Nature 1997, 385:729-733.

76. Moss ML, Jin SL, Milla ME, Bickett DM, Burkhart W, Carter HL, Chen WJ, Clay WC, Didsbury JR, Hassler D, et al.: Cloning of a disintegrin metalloproteinase that processes precursor tumour-necrosis factoralpha. Nature 1997, 385:733-736.

77. van Tetering $G$, van Diest $P$, Verlaan I, van der Wall E, Kopan $R$, Vooijs M The metalloprotease ADAM10 is required for notch1 S2 cleavage. J Biol Chem 2009, 284:31018-31027.

78. Bozkulak EC, Weinmaster G: Selective use of ADAM10 and ADAM17 in activation of Notch1 signaling. Mol Cell Biol 2009, 29:5679-5695.

79. Sahin U, Weskamp G, Kelly K, Zhou HM, Higashiyama S, Peschon J, Hartmann D, Saftig P, Blobel CP: Distinct roles for ADAM10 and ADAM17 in ectodomain shedding of six EGFR ligands. J Cell Bio/ 2004, 164:769-779.

80. Sapir A, Assa-Kunik E, Tsruya R, Schejter E, Shilo BZ: Unidirectional Notch signaling depends on continuous cleavage of Delta. Development 2005, 132:123-132.

81. X. tropicalis genome assembly v4.1 [http://genome.jgi-psf.org/Xentr4/ Xentr4.home.html]

82. Larkin MA, Blackshields G, Brown NP, Chenna R, McGettigan PA, McWilliam $\mathrm{H}$, Valentin F, Wallace IM, Wilm A, Lopez R, et al:: Clustal W and Clustal X version 2.0. Bioinformatics 2007, 23:2947-2948.
83. PHYLIP (Phylogeny Inference Package) version 3.6 [http:// evolution.genetics.washington.edu/phylip/]

84. Metazome [http://www.metazome.net/]

85. BLAT [http://genome.ucsc.edu/cgi-bin/hgBlat?command=start]

86. SignalP 3.0 [http://www.cbs.dtu.dk/services/SignalP

87. SMART [http://smart.embl-heidelberg.de]

88. Sive HL, Grainger RM, Harland RM: Early Development of Xenopus Laevis. A Laboratory Manual Cold Spring Harbor, Cold Spring Harbor Laboratory Press; 2000

doi: 10.1186/1471-2148-10-211

Cite this article as: Wei et al., Conservation and divergence of ADAM family proteins in the Xenopus genome BMC Evolutionary Biology 2010, 10:211

\section{Submit your next manuscript to BioMed Centra and take full advantage of:}

- Convenient online submission

- Thorough peer review

- No space constraints or color figure charges

- Immediate publication on acceptance

- Inclusion in PubMed, CAS, Scopus and Google Scholar

- Research which is freely available for redistribution

Submit your manuscript at www.biomedcentral.com/submit
C) Biomed Central 